\title{
Optimal adaptive control of (bio)chemical reactors: past, present and future
}

\author{
Ilse Y. Smets ${ }^{\text {a }}$, Johan E. Claes ${ }^{\text {a }}$, Eva J. November ${ }^{\mathrm{a}}$, \\ Georges P. Bastin ${ }^{\text {, }}$, Jan F. Van Impe ${ }^{\text {a,* }}$ \\ ${ }^{a}$ BioTeC-Bioprocess Technology and Control, Department of Chemical Engineering, Katholieke Universiteit Leuven, W. de Croylaan 46, \\ Leuven B-3001, Belgium \\ ${ }^{\mathrm{b}}$ CESAME, Université Catholique de Louvain, Louvain-la-Neuve B-1348, Belgium
}

\begin{abstract}
In this paper an overview of optimal adaptive control of (bio)chemical reactors is presented. Following the paradigm of the Minimum Principle of Pontryagin the derivation of optimal control sequences for fed-batch production processes is briefly revisited. Next, it is illustrated how the obtained optimal profiles can be exploited in the characterization of nearly optimal control sequences in terms of the qualitative behavior of the specific growth and production rates as function of the limiting substrates. Implementing this knowledge leads in a natural way to the design of (nearly) optimal adaptive feedback controllers. Special emphasis will lie on the potential of on-line biomass measurements (obtained with the Biomass Monitor) in the estimation algorithm of the growth kinetics being the adaptive component of the controller. Extensions towards fermentation processes with (i) multiple substrates and (ii) non-monotonic kinetics are also included. Finally, perspectives towards optimal adaptive control of not perfectly mixed (bio)chemical reactor systems, such as chemical tubular reactors, are outlined.
\end{abstract}

(C) 2004 Elsevier Ltd. All rights reserved.

Keywords: Minimum principle of Pontryagin; (Bio)chemical reactors; Adaptive control; Feedback control

\section{Introduction}

Given the recent re-orientation of some key players in the bulk chemical industry towards the field of life sciences in which fed-batch processes are predominant (e.g., production of baker's yeast, food additives and recombinant proteins), optimization and control of fedbatch bioreactors has become more challenging than ever. Fed-batch operation enables superior control as it provides different avenues in the substrate plane for control through distinct phases of biomass growth and metabolite production. By programming substrate feeding, one can control important phenomena such as substrate inhibition, glucose effect and catabolite repression. Furthermore, from the control engineering viewpoint, fed-batch processes are quite challenging, since the optimization of the substrate feed rate is a dynamic problem [26]. Unlike the continuously stirred 91

\footnotetext{
${ }^{*}$ Corresponding author. Tel.: +32-16-32-14-66; fax: +32-16-32-29-
}

tank reactors, the primary goal of a substrate feedback controller for a fed-batch fermentation process is not to stabilize the process globally, but rather to optimize it while keeping an inherently unstable type of behavior under control (see also [5]). Control opportunities in fedbatch operated fermentations have been reviewed in detail in a number of articles $[16,19,25,26]$. It is well known that the design of high-performance model-based control algorithms for biotechnological processes is hampered by two major problems which call for adequate engineering solutions. First, the process kinetics are most often poorly understood nonlinear functions, while the corresponding parameters are in general timevarying. Second, up till now there has been a lack of reliable sensors suited to real-time monitoring of the process variables which are needed in advanced control algorithms. Therefore, the earliest attempts at control of a biotechnological process used no model at all. Successful state trajectories from previous runs which had been stored in the process computer were tracked using open-loop control. Many industrial fermentations are still operated using this method. The need for the development of tracking controllers for the implementation 
of the (designed) open-loop profiles as well as the need for on-line recalculation of the profiles in case of (large) disturbances is apparent [2].

This article presents a unifying methodology for optimization of biotechnological processes, i.e., optimal adaptive control of which the rationale can be summarized as follows. Model-based optimal control studies (Minimum Principle of Pontryagin [17]) provide a theoretically realizable optimum. However, the real-life implementation will fail in the first place due to modeling uncertainties. On the other hand, model-independent adaptive controllers can be designed, but there is a priori no guarantee of the optimality of the results obtained. The gap between both approaches is bridged in two steps. First, heuristic control strategies are developed with nearly optimal performance under all conditions. These suboptimal controllers are based on biochemical knowledge concerning the process and on a careful mathematical analysis of the optimal control solution. In a second step, implementation of these profiles in an adaptive, model-independent way combines excellent robustness properties with nearly optimal performance. As an example, the design of a substrate feeding rate controller for a class of biotechnological processes in stirred tank reactors characterized by a decoupling between biomass growth and product formation is considered. A single as well as a multiple limiting substrate production process is covered. Furthermore, the concepts are applied to a non-perfectly mixed chemical conversion process in which space dependency has to be taken into account explicitly. It is shown that, under the simplifying assumptions of steady-state and plug flow behavior, optimization in space of tubular reactors is very similar to optimization in time of well-mixed reactors.

\section{General optimal control framework}

Optimization of fed-batch fermentations has been traditionally sought with respect to the volumetric substrate feed rate while keeping the substrate concentration in the feed fixed. Formulating the problem with the feed rate as the control variable yields a singular optimal control problem. Due to limited success in singular control theory, most of the fed-batch fermentation processes considered so far have been limited to loworder processes, described by no more than four mass balance equations (see, e.g., [15,31]). General computational algorithms have been developed by Lim et al. and Modak et al. for the optimal volumetric feed rate profiles for such fermentation processes [20,22].

Since most of the reported attempts to convert the singular control problem in a non-singular one (e.g., $[14,18,21,27,38])$ result in practical implementation problems, we adopt the traditional point of view, i.e., singular optimal control with the volumetric substrate feed rate as the manipulated variable.

As a case study, we consider processes with a decoupling between growth and production. It will be shown that the determination of the switching times in the feed rate profile can be simplified a lot by incorporating as much as possible microbiological/(bio)chemical knowledge concerning the process under consideration.

The case studies considered further in this paper belong to the following model class:

$\frac{\mathrm{d} \mathbf{x}(\zeta)}{\mathrm{d} \zeta}=\mathbf{f}[\mathbf{x}(\zeta)]+\mathbf{b} \cdot u \quad$ with $\zeta_{0}<\zeta<\zeta_{\mathrm{f}}$

with $\mathbf{x}(\zeta)$ the state vector and $u$ the scalar control input (both possibly restricted by physical constraints such as upper and lower bounds), and $\zeta$ the independent variable $t$ (time) or $z$ (the one-dimensional spatial coordinate). Subscripts 0 and $\mathrm{f}$ denote the initial and final value, respectively. As can be seen, the nonlinear model (1) is affine in the input $u$.

The performance criterion to be minimized is a sum of a terminal and an integral cost:

$J[u]=\underbrace{h\left[\mathbf{x}\left(\zeta_{\mathrm{f}}\right)\right]}_{\text {Terminal cost }}+\underbrace{\int_{0}^{\zeta_{\mathrm{f}}} g[\mathbf{x}(\zeta)] \mathrm{d} \zeta}_{\text {Integral cost }}$

Observe that performance measure (2) is assumed not to depend explicitly on the control input $u$. The problem statement is then the following:

Find an admissible control which causes the given system to follow an admissible trajectory while at the same time minimizing the performance criterion.

According to the Minimum Principle of Pontryagin [17] the Hamiltonian $\mathscr{H}$, equal to the sum of (i) the integrand of the integral cost and (ii) the product of the costate vector $\mathbf{p}$ with the right hand side of the balance equations, i.e.,

$$
\mathscr{H}=g[\mathbf{x}(\zeta)]+\mathbf{p}^{\mathrm{T}} \mathbf{f}[\mathbf{x}(\zeta)]+\mathbf{p}^{\mathrm{T}} \mathbf{b} \cdot u \triangleq \phi+\psi \cdot u
$$

must be minimized in order to minimize performance criterion (2).

The costate vector $\mathbf{p}$ satisfies the following system of differential equations:

$$
\begin{aligned}
& \frac{\mathrm{d} \mathbf{p}}{\mathrm{d} \zeta}\left(=-\frac{\partial \mathscr{H}}{\partial \mathbf{x}}\right) \\
& \quad=-\frac{\partial g[\mathbf{x}(\zeta)]}{\partial \mathbf{x}}-\mathbf{p}^{\mathrm{T}} \frac{\partial \mathbf{f}[\mathbf{x}(\zeta)]}{\partial \mathbf{x}} \quad \text { with } \mathbf{p}\left(\zeta_{\mathrm{f}}\right)=\left.\frac{\partial h}{\partial \mathbf{x}}\right|_{\zeta_{\mathrm{f}}}
\end{aligned}
$$

\subsection{Extremal control determination}

Because of the specific structure of model (1), a Hamiltonian function which is affine in the control input is obtained. As a result, the optimal control is of the 
bang-bang type, with the possibility of singular arcs depending on the value of $\psi$ :

- if $\psi>0$, then $u^{*}(\zeta)=u_{\mathrm{MIN}}$

- if $\psi=0$ for $z \in\left[\zeta_{i}, \zeta_{i+1}\right]$, then $u^{*}(\zeta)=u_{\text {sing }}(\zeta)$

- if $\psi<0$, then $u^{*}(\zeta)=u_{\mathrm{MAX}}$

$u_{\mathrm{MIN}}$ and $u_{\mathrm{MAX}}$ represent the lower and upper bound on the control input, respectively. Since the Hamiltonian does not explicitly depend on $\zeta$ (i.e., the time coordinate $t$ in a well mixed reactor case or the spatial coordinate $z$ in a plug flow reactor case), the Hamiltonian is constant when evaluated along an extremal trajectory.

\subsection{Singular arc analysis}

As previously mentioned, the term $\psi$ is equal to zero on a singular interval $\left[\zeta_{i}, \zeta_{i+1}\right]$, failing to provide, as such, an expression for the singular control law $u_{\text {sing }}(\zeta)$. The latter is then obtained by repeatedly differentiating $\psi$ with respect to $\zeta$ until $u$ appears explicitly.

Following some algebraic manipulations a general expression for the control on the singular arc can be found:

$u_{\text {sing }}(\zeta)=\frac{\left[\frac{\partial g[\mathbf{x}(\zeta)]}{\partial \mathbf{x}}+\mathbf{p}^{\mathrm{T}} \frac{\partial \mathbf{f}[\mathbf{x}(\zeta)]}{\partial \mathbf{x}}\right] \mathbf{d}-\left[\frac{\partial^{2} g[\mathbf{x}(\zeta)]}{\partial \mathbf{x}^{2}} \mathbf{b}+\mathbf{p} \frac{\partial \mathbf{d}}{\partial \mathbf{x}}\right] \mathbf{f}[\mathbf{x}(\zeta)]}{\left[\frac{\partial^{2} g[\mathbf{x}(\zeta)]}{\partial \mathbf{x}^{2}} \mathbf{b}+\mathbf{p}^{\mathrm{T}} \frac{\partial \mathbf{d}}{\partial \mathbf{x}}\right] \mathbf{b}}$

In this paper the concepts of optimal adaptive control will be illustrated with three case studies. The first two are fed-batch case studies focusing on optimization in time. The last case study is a tubular reactor process to illustrate optimization in space.

\subsection{Case studies}

\subsubsection{Optimization in time $(\zeta=t)$}

Fed-batch production processes relying on more than one limiting substrate, can be represented by following mass balance equations: ${ }^{1}$

$\overline{\mathrm{d}} \mathbf{\mathrm { d } t} \underbrace{\left[\begin{array}{c}S \\ N \\ X \\ P \\ V\end{array}\right]}_{\mathbf{x}(t)}=\underbrace{\left[\begin{array}{c}-\sigma_{S} X \\ -\sigma_{N} X \\ \mu X \\ \pi X-k P \\ 0\end{array}\right]}_{\mathbf{f}[\mathbf{x}(t)]}+\underbrace{\left[\begin{array}{cc}C_{S, \text { in }} & 0 \\ 0 & C_{N, \text { in }} \\ 0 & 0 \\ 0 & 0 \\ 1 & 1\end{array}\right]}_{\mathbf{b}} \underbrace{\left[\begin{array}{c}u_{S} \\ u_{N}\end{array}\right]}_{\mathbf{u}}$

$X$ [g DW] denotes the micro-organism, $S$ [g] the carbon source, $N[\mathrm{~g}]$ the nitrogen source and $P[\mathrm{~g}]$ the

\footnotetext{
${ }^{1}$ These balance equations easily simplify to a one limiting substrate case when the mass balance equation and the feeding of the second substrate, e.g., $N$, is neglected. Extension to more than two substrates is straightforward.
}

product. The input $u_{S}[\mathrm{~L} / \mathrm{h}]$ is the volumetric flow rate of the limiting carbon source while $u_{N}$ is the volumetric flow rate of the limiting nitrogen source. $V[\mathrm{~L}]$ is the volume of the reactor, and $C_{S, \text { in }}[\mathrm{g} / \mathrm{L}]$ and $C_{N \text {,in }}$ are the limiting carbon source concentration and limiting nitrogen source concentration in the feed, respectively. Further, $\sigma_{S}=\mu / Y_{X / S}+\pi / Y_{P / S}+m$ is the specific carbon source consumption rate (the extended linear law) with $Y_{X / S}$ [g DW/g] the yield coefficient of biomass on the carbon substrate, $Y_{P / S}$ the yield coefficient of product on substrate and $m[\mathrm{~g} /(\mathrm{g} \mathrm{DWh})]$ the maintenance constant. $\sigma_{N}=\mu / Y_{X / N}$ is the specific nitrogen source consumption rate with $Y_{X / N}[\mathrm{~g} \mathrm{DW} / \mathrm{g}]$ the yield coefficient of biomass on nitrogen. At this stage, the shape of the specific growth rate $\mu[1 / \mathrm{h}]$ and the specific production rate $\pi$ $[1 / \mathrm{h}]$ as function of the limiting concentrations is still arbitrary (i.e., monotonically increasing or non-monotonic). Finally, $k[1 / \mathrm{h}]$ is the product hydrolysis or degradation constant.

\subsection{Optimization in space $(\zeta=z)$}

In contrast with the above example of homogeneously mixed systems, a large amount of (bio)chemical processes is operated in (tubular) reactors where space dependency has to be taken into account explicitly. As a proto-type example of tubular reactor systems we focus on the most basic system, i.e., a steady-state plug flow reactor, surrounded by a cooling/heating jacket, in which an exothermic first order reaction takes place. The system is described by following energy and mass balance equations which fit in the framework of Eq. (1) in which $\zeta$ denotes the spatial variable $z$ [29].

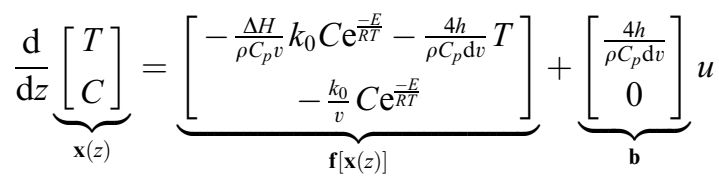

with $T(0)=T_{\text {in }}, C(0)=C_{\text {in }}$

$C, T$ and $u$ represent the reactant concentration, reactor temperature and the jacket fluid temperature (i.e., the manipulated variable), respectively. Explanation of the other symbols can be found in [29].

\section{Optimal control in time $(\zeta=t)$}

\subsection{Single substrate production process}

As an example of a single substrate $(S)$, growth decoupled production process, the penicillin $G$ production process is considered, in which the specific growth rate as well as the specific production rate (and thus $\sigma$ through the extended linear law relation) are assumed to be functions of substrate concentration $C_{S}$ only. As 
such, the mass balance equation for the nitrogen source $N$ as well as its inflow rate in Eq. (5) can be discarded. The specific growth rate $\mu\left(C_{S}\right)$ is monotonically increasing (Monod or Contois kinetics) and the specific production rate $\pi\left(C_{S}\right)$ is non-monotonically increasing (Haldane kinetics) as illustrated in Fig. 1.

When maximizing the final amount of product

$J[u]=-P\left(t_{\mathrm{f}}\right)$

with the final time $t_{\mathrm{f}}$ free and the total amount of carbon source that can be added fixed, Eq. (4) is translated into following optimal feed rate during the singular production phase $[35,36]$ :

$$
\begin{aligned}
u_{S, \text { sing }}(t)= & \frac{\sigma C_{X} V}{C_{S, \text { in }}-C_{S}}+k \\
& \times \frac{p_{3} V\left(\pi^{\prime} X-\mu^{\prime} P\right)}{X\left(C_{S, \text { in }}-C_{S}\right)\left(p_{1} \sigma^{\prime \prime}-p_{2} \mu^{\prime \prime}-p_{3} \pi^{\prime \prime}\right)}
\end{aligned}
$$

where a prime denotes derivation with respect to substrate concentration, and $p_{i}$ is the costate associated with component $x_{i}$ of the state vector $\mathbf{x}$. Note that this expression is linear in the specific product decay rate $k$, and is a feedback law of state variables only (it can be shown that the costates $p_{1}$ and $p_{2}$ depend linearly on $p_{3}$ ). Furthermore, the second term requires knowledge of an analytical expression of the derivatives of all specific rates up to second order.
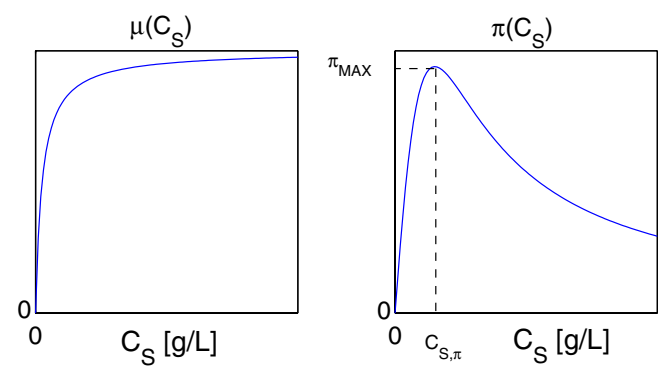

Fig. 1. Specific growth and production rates for penicillin G production process. Left plot: Monod type growth kinetics. Right plot: Haldane type production kinetics.
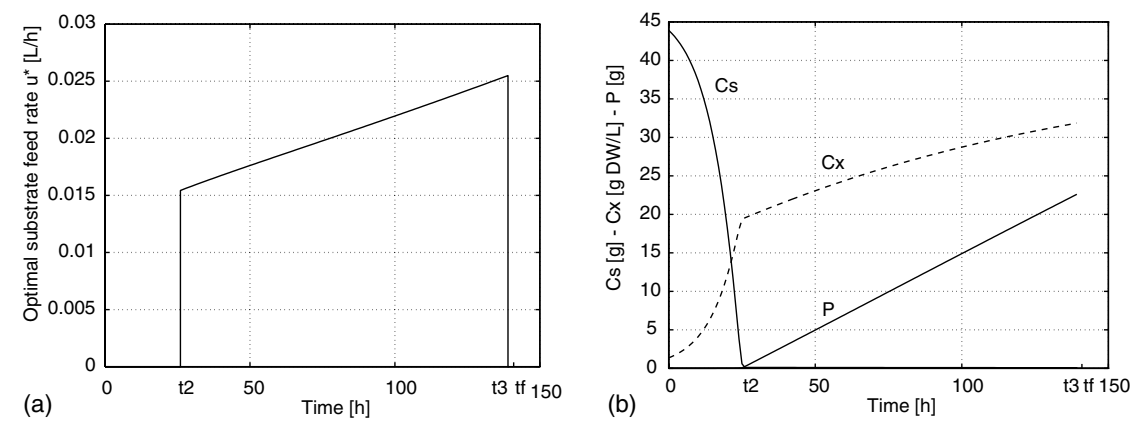

Fig. 2. Optimal control. Left plot: optimal substrate feed rate profile $u^{*}(t)$. Right plot: substrate concentration $C_{S}(t)$, biomass concentration $C_{X}(t)$, and product amount $P(t)$ profiles.
The optimal solution (unbounded control and state and free initial substrate concentration $C_{S}(0)$ ) for the penicillin fed-batch fermentation case study is summarized in Fig. 2 and will be further explained below. The parameter and numerical simulation values can be found in [8].

\section{Heuristic control strategies in time $(\zeta=t)$}

\subsection{Single substrate production process}

The most important drawbacks of the optimal control solution as outlined above for the single substrate production process can be summarized as follows:

(1) Optimal control is a very model sensitive technique. It requires a complete knowledge of the process model, including an analytic expression for all specific rates. Since in biotechnology this assumption is in practice never fulfilled, the optimal profile is generally calculated using a highly simplified model describing the process more or less correctly only from a qualitative view-point. Therefore, the resulting optimal profiles can be used only to increase the insight into both the process and the quality of the model.

(2) For the maximization of the final product amount $P\left(t_{\mathrm{f}}\right)$, the optimal feed rate profile is obtained in complete state feedback form except for the switching time $t_{2}$ between the batch growth phase and the singular production phase (Fig. 2). In general, $t_{2}$ must be determined numerically in advance.

(3) Necessary and sufficient conditions can be derived for which $t_{2}$ also becomes a function of state variables only [33]. However, even if a perfect process model could be available which satisfies all conditions to obtain the complete optimal solution in closed loop, real-life implementation is still hampered by the lack of reliable sensors suited to realtime monitoring of the process variables needed in the controller. Besides a perfect analytical knowl-

(b) 
edge of all specific rates and corresponding parameters, the control during the singular phase $u_{\text {sing }}(t)$ requires on-line measurements of all state variables $S$, $X, P$, and $V[35,36]$.

Therefore, it is very useful to construct suboptimal strategies that do not suffer from the above difficulties, at the expense of as small as possible a decrease in performance. In [35-37] nearly optimal heuristic controllers for both the substrate concentration $C_{S}$ (heuristic $C_{S}$-control) and the overall specific growth rate $\mu$ (heuristic $\mu$-control) are designed. As an example, in this paper heuristic $C_{S}$-control is considered, which can be motivated from both the microbiological and mathematical point of view.

\subsubsection{Microbiological and experimental motivation}

The construction of a nearly optimal profile for the type of biotechnological processes under consideration can be based on the concept of a biphasic fermentation.

(1) Growth phase $\left[0, t_{2}\right]$. During the growth phase the specific substrate-to-biomass conversion rate $\mu$ is focused. For the control needed reference is made to the optimal control results: in the case of an unbounded input $u_{S}$, an unconstrained state vector $\mathbf{x}$, and a free initial substrate concentration $C_{S}(0)$, the growth phase is a batch phase. A general strategy is that the fraction $\alpha_{\text {growth }}$ of the total amount of substrate available $\alpha$, which is consumed for biomass accumulation during the growth phase, must be added as fast as possible in order to obtain the highest possible value of $\mu$.

(2) Production phase $\left[t_{2}, t_{3}\right]$. During production the specific production rate $\pi$ is focused. As shown in Fig. $1, \pi$ exhibits a maximum as a function of the substrate concentration $C_{S}$. So, it is a reasonable control objective to keep the substrate concentration during the production phase constant at the level $C_{S, \pi}$ which maximizes $\pi$. For instance, in the case of Haldane kinetics, $C_{S, \pi}$ equals $\left(K_{P} K_{I}\right)^{1 / 2}$. Therefore, as soon as $C_{S}(t)$ equals $C_{S, \pi}$, the feed rate switches from $u_{S}(t)=0$ to

$u_{S, \text { production }}=\frac{\sigma C_{X} V}{C_{S, \text { in }}-C_{S}}$

which keeps substrate concentration $C_{S}$ constant during production. Controller (9) is shut off when all substrate $\alpha$ has been added at time $t=t_{3}$, or equivalently, when $V\left(t_{3}\right)=V_{\mathrm{f}}$. As in the case of optimal control, the fermentation continues in batch $[u(t)=$ $\left.0, t_{3}<t<t_{\mathrm{f}}\right]$ until the net product formation rate $\mathrm{d} P / \mathrm{d} t$ equals zero at $t=t_{\mathrm{f}}$.

Obviously, the switching time $t_{2}$ (Fig. 2) is known in closed loop: the production phase starts when the sub- strate concentration $C_{S}$ becomes equal to $C_{S, \pi}$. As a result, the optimization problem has been reduced to the one-dimensional optimization of the initial substrate concentration $C_{S}(0)$, or more generally, of the fraction $\alpha_{\text {growth }}$ of the total substrate amount available.

A further refinement of this strategy consists of optimizing the value of the substrate concentration level during production (denoted by $C_{S}^{*}$, which plays the role of a setpoint). In other words, during production $C_{S}$ is kept constant, but not necessarily at the value $C_{S, \pi}$ which maximizes $\pi$. As in the case of optimal control, optimization of final product amount reduces to a two-dimensional optimization problem. The degrees of freedom are the initial substrate concentration $C_{S}(0)-o r$ more generally, the fraction $\alpha_{\text {growth }}$ and the substrate concentration setpoint $C_{S}^{*}$ during production. Heuristic control strategies for other combinations of monotonic and non-monotonic growth and production kinetics, can be found in [8].

\subsubsection{Mathematical justification}

With respect to Eq. (8) it is shown in $[35,36]$ that the proposed heuristic $C_{S}$-controller reduces to the optimal profile if (and only if) (i) the performance index is independent of final time $t_{\mathrm{f}}$, (ii) the specific rates $\mu$ and $\pi$ are functions of $C_{S}$ only, (iii) $k=0$, and (iv) the production phase starts when the substrate concentration $C_{S}$ reaches the level which maximizes the ratio $\pi / \sigma$. In cases where (some of) these conditions are not satisfied, the proposed heuristic $C_{S}$-controller is at least a very good approximation of the optimal solution.

\subsection{Multiple substrate production process}

To illustrate optimization of a multiple substrate fedbatch process, the production of PHB (poly- $\beta$ hydroxybutyric) acid in an aerobic culture of Alcaligenes eutrophus is considered. The mathematical model (presented in Eq. (5)) is based on following a priori knowledge. Two limiting substrates are needed for the microbial growth: fructose as carbon source $S$ and ammonia as nitrogen substrate $N$. The intracellular production of PHB acid, by fructose degradation, can take two different paths: the first being associated with the growth but with a very small yield (and therefore neglected in this case study); the second being enzyme catalyzed and completely inhibited by nitrogen $N$. The shape of the specific rates for growth $\mu$ and production $\pi$ as function of the two limiting substrates is shown in Fig. 3. Endogenous metabolism is neglected.

The aim is again to maximize the final amount of product $P$ by manipulating the incoming flow rate, i.e., both flow rates $u_{S}$ and $u_{N}$ in this case study. The final time $t_{\mathrm{f}}$ is free and the total amount of carbon $S$ and nitrogen $N$ is fixed. 

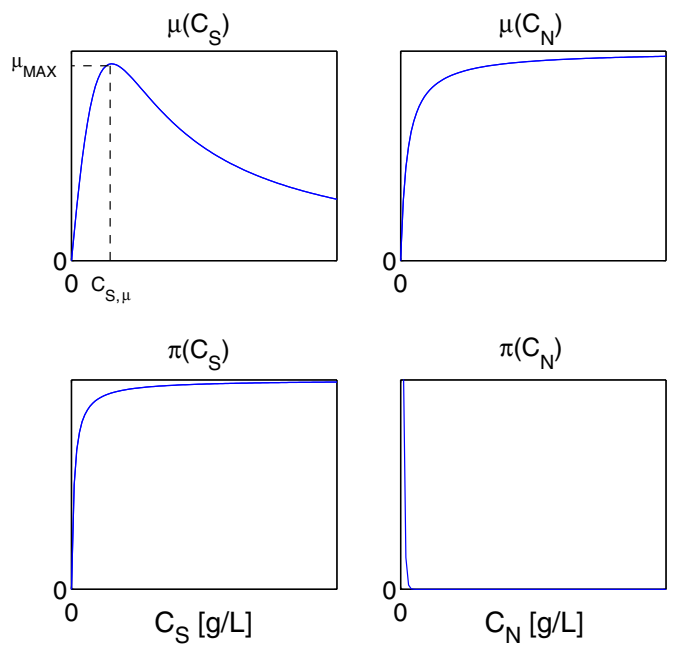

Fig. 3. Specific growth and production rates as function of the limiting substrates for the PHB fed-batch production process.

Since the process occurs in two phases (i.e., a growth phase followed by a production phase) following heuristic strategy will be close to optimal:

(1) Growth phase

- $u_{N}$ : add all nitrogen available at time $=0$, since $\mu\left(C_{N}\right)$ increases monotonically with $C_{N}$.

- $u_{S}$ : keep the carbon source concentration $C_{S}$ constant at some reference level $C_{S}^{*}$. An appropriate initial guess is $C_{S}^{*}=C_{S, \mu}$, the concentration which maximizes $\mu\left(C_{S}\right)$.

(2) Production phase

- $u_{N}=0$ since PHB synthesis is inhibited by $N$.

- $u_{S}$ : add the remaining amount of fructose as fast as possible, since $\pi\left(C_{S}\right)$ increases monotonically with $C_{S}$.

Obviously, the switch between both phases occurs as soon as nitrogen $N$ has been completely exhausted. The process is stopped as soon as $\mathrm{d} P / \mathrm{d} t=0$.

Extension towards more than two limiting substrates can be found in [34].

\section{Linearizing control in time $(\zeta=t)$}

With respect to a real-life implementation, the heuristic controller (9) has the following advantages over the optimal controller (8). First, the switching time $t_{2}$ between growth and production (and thus the complete control) is known in closed loop as a function of the state: $C_{S}\left(t_{2}\right)=C_{S}^{*}$. Second, as for the modeling uncertainty problem, only the specific substrate consumption rate $\sigma$ is required. Third, as for the on-line monitoring problem, the number of state variables to be measured on-line has been reduced by one: there is no need for a measurement of the product $P$. This is an important advantage in cases where the product remains (almost) completely in the liquid phase of the reactor. Finally, the most important advantage is that the given optimal control problem-namely, optimization of the final product amount $P\left(t_{\mathrm{f}}\right)$ at some unknown final time $t_{\mathrm{f}}$ has been replaced by a more common regulator problem-namely, regulation of substrate concentration $C_{S}$ to some setpoint $C_{S}^{*}$ for all time $t$ during productionfor which feedback control loops can be developed.

However, a real-life implementation is still far away. Two important problems remain to be solved.

Problem 1 (The monitoring problem). Although the number of unknowns has been reduced, the heuristic $C_{S^{-}}$ controller still needs on-line measurements - or at least reliable estimates - of substrate $S$, biomass $X$, volume of the liquid phase $V$, and of the specific substrate consumption rate $\sigma$.

Problem 2 (The stability problem). The closed loop stability is not guaranteed a priori. From the general model (5) the closed loop dynamics during production for substrate concentration $C_{S}$ when using controller (9) are simply:

$\frac{\mathrm{d} C_{S}}{\mathrm{~d} t}=0$

Clearly, even a small disturbance can move substrate concentration irreversibly away from its desired value $C_{S}^{*}$, resulting in performance degradation.

In the following it is illustrated how to design controllers based on the heuristic approach that do not suffer from the above drawbacks.

\subsection{The stability problem}

The second problem is considered first. When replacing the optimal controller (8) by the heuristic controller (9) the control objective becomes more realistic, namely setpoint control or more generally tracking of a reference profile. The heuristic controller (9) performs well if there are no disturbances, measurement errors, etc., and if the switch from growth to production occurs exactly when $C_{S}\left(t_{2}\right)=C_{S}^{*}$. As in general these assumptions are not fulfilled, some mechanism must be incorporated in control law (9) which controls the tracking error. At this point the principle of linearizing control can be used. An introduction and several applications in bioreactor control can be found in [5] and the references therein.

(1) Focusing again on the one limiting substrate fedbatch case study, the control variable is the volumetric feed rate $u_{S}$, while the controlled variable is the 
substrate concentration $C_{S}$. So an inputloutput model for this case is simply the differential equation of the substrate concentration:

$\frac{\mathrm{d} C_{S}}{\mathrm{~d} t}=-\sigma C_{X}-C_{S} \frac{u_{S}}{V}+C_{S, \text { in }} \frac{u_{S}}{V}$

This input/output model (which is linear in the control $u_{S}$ ) is of relative degree one: the control $u_{S}$ appears explicitly in the first derivative (with respect to time $t$ ) of the controlled variable $C_{S}$.

(2) A linear stable ( $\tau$ is a strictly positive given number) reference model for the tracking error is then

$\frac{\mathrm{d}\left(C_{S}-C_{S}^{*}\right)}{\mathrm{d} t}=-\tau\left(C_{S}-C_{S}^{*}\right)$

Note that the reference model is of the same degree as the input/output model. At this point, the reference signal $C_{S}^{*}$ may be time-varying.

(3) A nonlinear linearizing controller is obtained by eliminating $\mathrm{d} C_{S} / \mathrm{d} t$ between (10) and (11):

$u_{0}=\frac{\sigma C_{X}+\frac{\mathrm{d} C_{S}^{*}}{\mathrm{~d} t}-\tau\left(C_{S}-C_{S}^{*}\right)}{C_{S, \text { in }}-C_{S}} V$

In the application considered here, a constant substrate concentration during the production phase is desired, so:

$u_{0}=\frac{\sigma C_{X} V}{C_{S, \text { in }}-C_{S}}-\tau \frac{\left(C_{S}-C_{S}^{*}\right)}{C_{S, \text { in }}-C_{S}} V$

In most practical situations the control action (i.e., the feeding pump capacity) is bounded. The resulting controller during the production phase is then:

$u_{S, \text { production }}= \begin{cases}u_{0} & \text { if } 0 \leqslant u_{0} \leqslant U_{\mathrm{MAX}} \\ 0 & \text { if } u_{0} \leqslant 0 \\ U_{\mathrm{MAX}} & \text { if } u_{0} \geqslant U_{\mathrm{MAX}}\end{cases}$

Remark 1. Obviously, this controller reduces to heuristic controller (9) if the tracking error $\left(C_{S}-C_{S}^{*}\right)$ equals zero.
Remark 2. As motivated in [35], an important advantage over the heuristic controller (9) is that controller (13) can be implemented from $t=0 \mathrm{on}$, by considering $C_{S}^{*}$ as the setpoint from $t=0$ on.

The above is illustrated for the penicillin G fed-batch fermentation in Fig. 4 where it is assumed that the measurements are all perfect and that there are no disturbances. Observe that the nonlinear linearizing controller (13) becomes positive before $C_{S}$ reaches $C_{S}^{*}$. This guarantees a smooth transition in the substrate concentration profile at the start of the production phase. This behavior can be obtained using a small value of $\tau$ (e.g., $\tau=1$ ) in the reference model (11). On the other hand, a large value of $\tau$ increases the stability margin, tracking behavior and disturbance rejection. This can be easily seen when calculating the closed loop response of substrate concentration $C_{S}$. Since $\tau$ is at the disposal of the user, it can be used to search for an optimal trade-off.

\subsection{The monitoring problem}

In the following sections the first problem is considered, i.e., monitoring of all variables required in controller (13). Possible solutions depend on which measurements are available on-line. The remaining variables are then estimated on-line using software sensors. The here presented algorithm is based on the minimal modeling concept introduced and discussed in, e.g., [5,12]. In this approach no assumption is made concerning the exact analytical structure of the specific rates required in the control law, thus circumventing the modeling and corresponding parameter identification problem. Instead, they are treated as time-varying parameters which are estimated on-line. By doing so, the nonlinear linearizing controller (13) is made adaptive and can be implemented independently of the - usually unknown - analytical expression for the specific rates.

While adaptive control based on on-line measurements of the substrate concentration $C_{S}$ is treated in,
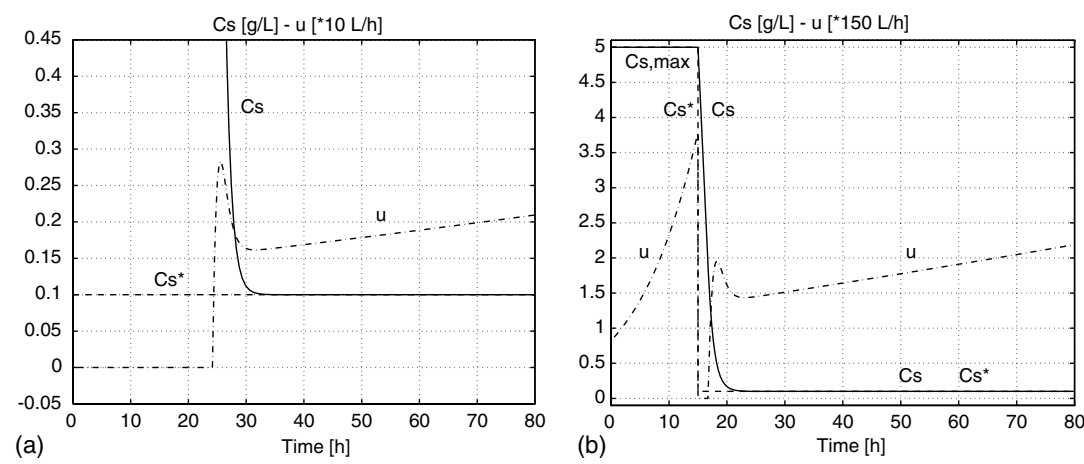

Fig. 4. Nonlinear linearizing control. Left plot: no constraints. Right plot: inequality constraint on $C_{S}$. (-) Actual substrate concentration $C_{S}$; (----) reference profile $C_{S}^{*} ;(-\cdot \cdot)$ control action $u$ (i.e., $\left.u_{S}\right)$. 
e.g., [35], we will focus here on the availability of on-line biomass concentration measurements.

\subsubsection{Biomass concentration measurements}

On-line measurements of the biomass concentration are provided in our research group by the Biomass Monitor (BM 214-M, Aber Instruments LTD, Aberystwyth, UK). This device measures the dielectric permittivity of microbial suspensions. Application of an electric field in a cell suspension causes a shift in the ions present resulting in a polarization of the cell membrane which behaves as a capacitor. Consequently, the capacitance is a measure of the induced charge separation due to the presence of viable cells. Dead cells or cells with ruptured membranes do not add to the capacitance signal [11]. This capacitance signal has proven to be a reliable measurement during several fermentations including yeast experiments (with baker's yeast [9] and Candida utilis [24]) as well as bacterial cell cultures (e.g., activated sludge [23]).

\subsubsection{Specific growth rate observer}

Following state-observer-based estimator for the specific growth rate is proposed (for more details reference is made to [5]):

$$
\begin{aligned}
& \frac{\mathrm{d} \widehat{C_{X}}}{\mathrm{~d} t}=\hat{\mu} C_{X}-\frac{u}{V} C_{X}-\omega\left(C_{X}-\widehat{C_{X}}\right) \\
& \frac{\mathrm{d} \hat{\mu}}{\mathrm{d} t}=\gamma C_{X}\left(C_{X}-\widehat{C_{X}}\right)
\end{aligned}
$$

where $\mathrm{a}^{\wedge}$ denotes an estimated quantity and $\omega[1 / \mathrm{h}]$ and $\gamma\left[\mathrm{L}^{2} /\left(\mathrm{g}^{2} \mathrm{~h}^{2}\right)\right]$ are tuning functions. When selecting these tuning functions equal to $\omega=2 \lambda[1 / \mathrm{h}]$ and $\gamma=\lambda^{2} / C_{X}^{2}$, the dynamics of the corresponding error system are governed by the (double) eigenvalue $-\lambda$. Fine-tuning of the observer reduces to selecting a suitable value for $\lambda$ to ensure a trade-off between convergence rate and noise sensitivity. As such, the tuning of this so-called Bastin and Dochain observer is comparable with the tuning of the high-gain observers proposed by [13], which are in fact only differing slightly in terms of the expression for the tuning parameter $\gamma$. Alternative observers, such as an Extended Kalman Filter (EKF) and the so-called robust $P I^{2} \mu$-observer of [1] are not considered here. The implementation of the former, although frequently proposed, is quite complex without guaranteeing stability and convergence within finite time. The latter is not a valid alternative due to the need for on-line measurements of the substrate concentration which are assumed not to be available in this study.

\subsubsection{Experimental validation of the specific growth rate observer}

5.2.3.1. Tuning of the observer. Experimental validation of the $C_{X}$ based $\mu$-observer is reported in $[9,10,24]$. To enhance the real-life performance several modifications are proposed. The first modification is introduced to avoid negative values of the estimated specific growth rate when the biomass concentration signal is low. Different strategies are possible to tackle this problem. The lowering of the $\gamma$ tuning factor by introduction of a constant factor $C_{X, c}$ is one possible solution. $\gamma$ is then equal to $\lambda^{2} /\left(C_{X, c}+C_{X}^{2}\right)$. Another option relies on setting bounds for $\hat{\mu}$. During the experimental validation of the specific growth rate estimator for the growth of Candida utilis [24] the first option was to be preferred with $C_{X, c}$ equal to $4 \mathrm{pF}^{2}$. Secondly, the choice of the optimal tuning factor and the initial value of the estimated specific growth rate, have proven to be of major importance for the convergence of the estimated value to the true value of the specific growth rate. Higher tuning factors are preferred in order to achieve fast convergence of the estimation and diminish the impact of incorrectly defined initial estimated specific growth rate values. For higher tuning factors, however, it is advisable to use filtered capacitance data in the estimator to prevent results with very unrealistic values of the specific growth rate. In [24] a Butterworth filter with a cutoff frequency of $0.0005 \mathrm{~Hz}$ and order 1 is proposed while in [9] the use of a second order Butterworth filter is illustrated.

\subsubsection{Monotonic and non-monotonic growth kinetics.} While assuming that on-line measurements of the substrate concentration are not available, but only on-line measurements of the biomass concentration $C_{X}$ (and, based on these, on-line estimates of the specific growth rate $\mu$ ) can be supplied, a control law similar to control law (12) can be derived. The control objective is reformulated from reaching a desired setpoint for the substrate concentration $C_{S}^{*}$ to reaching a desired setpoint for the specific growth rate $\mu^{*}$ (note that keeping the specific growth rate at a certain setpoint can also be the prime objective):

$u=\frac{\sigma C_{X}}{C_{S, \text { in }}-C_{S}^{*}} V-\tau_{\mu} \frac{\mu-\mu^{*}}{C_{S, \text { in }}-C_{S}^{*}} V$

with $\tau_{\mu}[\mathrm{g} / \mathrm{L}]$ a strictly positive tuning factor.

In case of monotonic kinetics for $\mu$, controller (15) performs well as illustrated in Fig. 5. After a start-up phase (not shown) control law (15), combined with the $C_{X}$ based $\mu$-observer (14), is able to keep the process, i.e., a growth process of Saccharomyces cerevisiae in a $5 \mathrm{~L}$ computer controlled bioreactor, at the desired setpoint of $0.12 \mathrm{1} / \mathrm{h}$. Even the jump to a different setpoint (i.e., $0.15 \mathrm{l} / \mathrm{h}$ ) after $850 \mathrm{~min}$ is tracked. Note that the specific growth rate for baker's yeast is generally assumed to be a non-monotonically increasing kinetics. However, the species dealt with here, showed only very moderate inhibition effects.

If inhibition effects cannot be neglected, non-monotonic kinetics have to be dealt with. A setpoint of the specific growth rate $\mu^{*}$ corresponds then with two values 


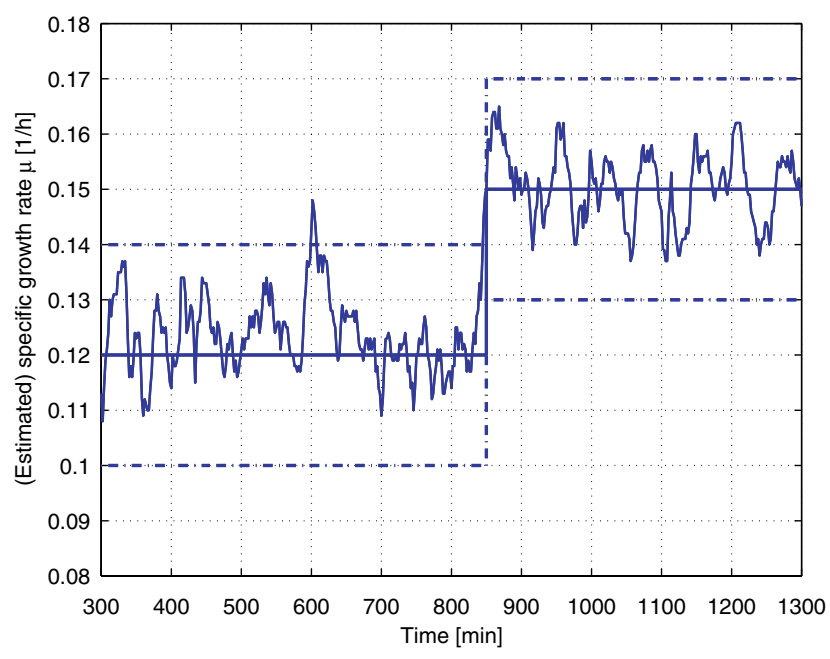

Fig. 5. Fed-batch growth of Saccharomyces cerevisiae. Experimental validation of control law (15) combined with the specific growth rate observer (14) and Biomass Monitor measurements. Specific growth rate setpoint $\mu^{*}$ switches from 0.12 to $0.151 / \mathrm{h}$ after $850 \mathrm{~min}$.

for the substrate concentration, i.e., the desired setpoint $C_{S}^{*}$ and a so-called associated substrate concentration $C_{S, \mathrm{a}}^{*}$. Therefore, mechanisms must be incorporated to assure the convergence to the desired setpoint. As explained in [28], this can be done by introducing a switch factor.

$u=\frac{\sigma C_{X}}{C_{S, \text { in }}-C_{S}^{*}} V-\tau_{\mu} f \frac{\mu-\mu^{*}}{C_{S, \text { in }}-C_{S}^{*}} V$

The switch factor $f$ is equal to +1 or -1 depending on (i) the positioning of the desired setpoint $C_{S}$ with respect to the $C_{S, \mu}$ value and (ii) the evolution of the specific growth rate (i.e., increasing or decreasing) as is presented in the flow chart of Fig. 6.

\section{Optimal control in space $(\zeta=z)$}

The case studies that have been treated so far are assumed to occur in perfectly mixed reactors. The hypothesis of homogeneously mixed systems is however not always valid. A large amount of (bio)chemical processes is operated in (tubular) reactors where space dependency has to be taken into account explicitly. Due to the increased complexity of such processes, analytical optimal control solutions are very scarce $[7,32]$ and one mostly relies on numerical optimization (e.g., with control vector optimization techniques [6]). Under the simplifying assumption of steady-state plug flow behavior, analytical control solutions for a tubular reactor as described by the energy and mass balance equation (6) are presented in [29]. Obviously, the optimization task shifts now from the time to the space domain, but, as outlined in [30], generic properties for the optimization in both domains exist.

As an illustration, following cost criterion is considered:

$$
\begin{aligned}
J[u] & =(1-A) J_{1}[u]+A J_{2}[u] \\
& \triangleq(1-A)[C(L)]+A \int_{0}^{L} \frac{\left[T(z)-T_{\text {in }}\right]^{2}}{K} \mathrm{~d} z
\end{aligned}
$$

with $A[-]$ the trade-off coefficient between terminal and integral cost and $T_{\text {in }}[\mathrm{K}]$ the fluid inlet temperature. $K$ is a constant (set equal to 250,000), introduced to equalize the order of magnitude of the two terms.

The terminal cost part minimizes the concentration of the reactant at the end of the tubular reactor and is therefore a measure for the process efficiency. The integral cost part penalizes the deviation of the reactor temperature $T$ from the inlet temperature to minimize the risk of temperature run-away and/or formation of hot spots.

From the general analysis of the extremal control sequences presented in the first sections it is clear that the control will be of the bang-singular-bang type.

Since in this example the integral cost part $g(\mathbf{x}(z))$ is not a function of the reactant concentration $C$, Eq. (4) reduces to following singular control:

$u_{\text {sing }}(z)=T+\frac{\Delta H d}{4 h} k_{0} C \mathrm{e}^{-\frac{E}{R T}}$

At this stage, the optimal control law is an open loop control law associated to which are model uncertainty and monitoring problems as mentioned before. The combination of the singular control expression with the

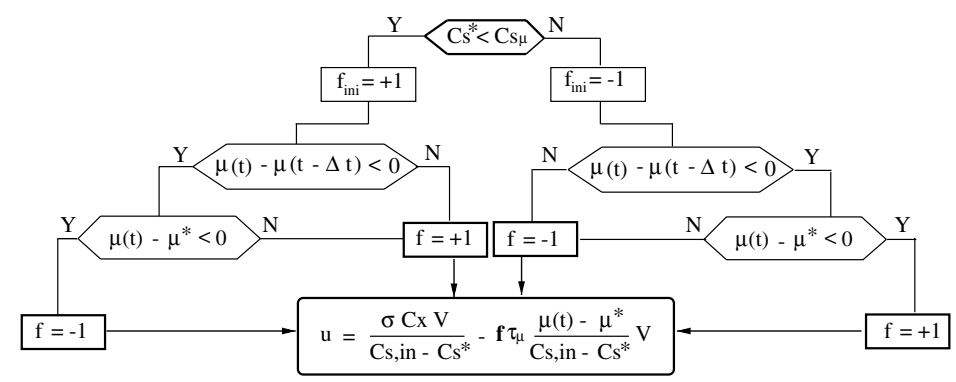

Fig. 6. Non-monotonic growth kinetics: decision algorithm for switch factor $f$ in control law (16). 
Table 1

Optimal adaptive control: a unifying approach

\begin{tabular}{cl}
\hline $\begin{array}{l}\text { Optimal control } \\
\downarrow\end{array}$ & model sensitive, open loop \\
microbiological/biochemical process knowledge & mathematical analysis of the optimal control solution \\
mouristic control & model-independent control objective \\
$\downarrow$ & linearizing control \\
Optimal adaptive control & adaptive state and parameter estimation robust, nearly optimal \\
& performance \\
\hline
\end{tabular}

balance equation of the temperature (Eq. (6)) reveals, however, that this control keeps the reactor temperature constant along the singular arc. Again a reference profile is provided for which adaptive tracking controllers can be designed.

\section{Discussion and conclusions}

To conclude this paper it can be stated that the framework of optimal adaptive control (summarized in a schematic way in Table 1 still offers interesting perspectives to obtain robust and practically realizable, nearly optimal control solutions for (bio)chemical conversion processes. The design consists of the following steps:

Step 1. Derivation of the optimal control solution to the given optimization problem, under the assumption of a perfectly known process model.

Step 2. Derivation of nearly optimal heuristic controllers, based on a careful analysis of the optimal control solution of Step 1 from both the biochemical and the mathematical point of view. This second step itself consists of:

(1) Detection of process variables which characterize the optimal control solution, such as a concentration or a specific rate.

(2) Construction of a reference profile for the characteristic process variable as a function of time.

As such, the optimization problem of Step 1 is replaced by a more common tracking control problem, for which feedback control loops are designed in Step 3.

Step 3. Nonlinear adaptive implementation of the derived heuristic controller in two steps:

(1) Embedding of the heuristic controller within a nonlinear linearizing controller.

(2) Adaptive estimation of the states and parameters which are not available on-line. According to the minimum modeling principle, no assumption is made concerning the exact analytic nature of the specific rates needed in the control algorithm.

Apart from the in this paper described indirect optimization method, a lot of progress has been made in direct optimization methods which transform the original infinite dimensional problem into a finite nonlinear programming (NLP) problem by either complete parameterization of the state and control vectors or parameterization of control vectors only (i.e., CVP techniques). Although these methods lead to case specific solutions since they rely on numerical optimization strategies, their power must not be underestimated. Banga and coworkers provide an up-to-date overview of the existing methods for bioreactor optimization [3]. As they illustrate, the performance of hybrid methods, combining the advantages of deterministic and stochastic methods, is remarkable.

Given the complexity of the majority of todays' industrial applications, straightforward analytical results from, e.g., the Minimum Principle of Pontryagin are hard to obtain. Therefore, we believe the future of optimal control of (bio)chemical production processes, will rely on a combination of direct and indirect optimization methods. If a simplified representation of the process exists for which an analytical result can be obtained with an indirect approach, these optimal profiles can serve as a starting point for numerical direct optimization algorithms when the complexity of the process representation is increased. A similar approach for parameter identification for (bio)chemical processes proved already successful in the past [4] and ongoing research along these lines (with respect to control) will be reported by the authors in the future.

\section{Acknowledgements}

Ilse Smets is a postdoctoral fellow with the Fund for Scientific Research Flanders (FWO). Work supported in part by Project OT/99/24 of the Research Council of the Katholieke Universiteit Leuven and the Belgian Program on Interuniversity Poles of Attraction, initiated by the Belgian State, Prime Minister's Office for Science, Technology and Culture. The scientific responsibility is assumed by its authors.

\section{References}

[1] R. Aguilar, J. González, M. Barrón, R. Martínez-Guerra, R. Maya-Yescas, Robust $\mathrm{PI}^{2}$ controller for continuous bioreactors, Process Biochemistry 36 (2001) 1007-1013.

[2] J. Banga, A. Alonso, R. Singh, Stochastic dynamic optimization of batch and continuous bioprocesses, Biotechnology Progress 13 (3) (1997) 326-335. 
[3] J. Banga, E. Balsa-Canto, C. Moles, A. Alonso, Dynamic optimization of bioreactors: a review, Proceedings of the Indian Academy of Sciences 69A (3-4) (2003) 257-265.

[4] J. Banga, K. Versyck, J. Van Impe, Numerical strategies for optimal experimental design for parameter identification of nonlinear dynamic (bio-)chemical processes, in: Proceedings of ESCAPE-10, 10th European Symposium on Computer Aided Process Engineering, Florence, Italy, 2000.

[5] G. Bastin, D. Dochain, On-line Estimation and Adaptive Control of Bioreactors, Elsevier Science Publishing, Amsterdam, 1990.

[6] G. Buzzi-Ferraris, E. Facchi, P. Forzatti, E. Tronconi, Control optimization of tubular catalytic reactors with catalyst decay, Industrial \& Engineering Chemistry Process Design and Development 23 (1984) 126-131.

[7] A. Chou, W. Ray, R. Aris, Simple control policies for reactors with catalyst decay, Transaction of the Institution of Chemical Engineers 45 (1967) T153-T159.

[8] J. Claes, A. Geeraerd, J. Van Impe, Heuristic feed rate profiles for optimal yield and productivity of fed-batch bioprocesses, Chemical Engineering Communications 172 (1999) 189-216.

[9] J. Claes, J. Van Impe, On-line estimation of the specific growth rate based on viable biomass measurements: experimental validation, Bioprocess Engineering 21 (5) (1999) 389-395.

[10] J. Claes, J. Van Impe, Combining yield coefficients and exit-gas analysis for monitoring of the baker's yeast fed-batch fermentation, Bioprocess Engineering 22 (2000) 195-200.

[11] C. Davey, The Biomass Monitor Source Book. A detailed user guide, Aber Instruments, Aberystwyth, Wales, UK, 1993.

[12] D. Dochain, M. Perrier, Dynamical modelling, analysis, monitoring and control design for nonlinear bioprocesses, Advances in Biochemical Engineering/Biotechnology 56 (1997) 147-197.

[13] M. Farza, S. Othman, H. Hammouri, J. Biston, A nonlinear approach for the on-line estimation of the kinetic rates in bioreactors: application to a lactic acid production process, Bioprocess Engineering 17 (1997) 143-150.

[14] R. Guthke, W. Knorre, Optimal substrate profile for antibiotic fermentations, Biotechnology and Bioengineering 23 (1981) 27712777.

[15] J. Hong, Optimal substrate feeding policy for a fed batch fermentation with substrate and product inhibition kinetics, Biotechnology and Bioengineering 28 (1986) 1421-1431.

[16] A. Johnson, The control of fed-batch fermentation processes - a survey, Automatica 23 (6) (1987) 691-705.

[17] D. Kirk, Optimal Control Theory: An Introduction, PrenticeHall, Englewood Cliffs, NJ, 1970.

[18] Z. Kurtanjek, Optimal nonsingular control of fed-batch fermentation, Biotechnology and Bioengineering 37 (1991) 814-823.

[19] J. Lee, S. Lee, S. Park, A. Middelberg, Control of fed-batch fermentations, Biotechnology Advances 17 (1999) 29-48.

[20] H. Lim, Y. Tayeb, J. Modak, P. Bonte, Computational algorithms for optimal feed rates for a class of fed-batch fermentation: numerical results for penicillin and cell mass production, Biotechnology and Bioengineering 28 (1986) 1408-1420.

[21] J. Modak, H. Lim, Simple nonsingular control approach to fedbatch fermentation optimization, Biotechnology and Bioengineering 33 (1989) 11-15.
[22] J. Modak, H. Lim, Y. Tayeb, General characteristics of optimal feed rate profiles for various fed-batch fermentation processes, Biotechnology and Bioengineering 28 (1986) 1396-1407.

[23] E. November, J. Van Impe, On-line viable biomass measurements and estimation of the specific growth rate of activated sludge from municipal wastewater treatment, Water Science and Technology 43 (7) (2001) 97-102.

[24] E. November, J. Van Impe, The tuning of a model-based estimator for the specific growth rate of Candida utilis, Bioprocess and Biosystems Engineering 25 (2002) 1-12.

[25] S. Parulekar, H. Lim, Modeling, optimization and control of semi-batch bioreactors, Advances in Biochemical Engineering/ Biotechnology 32 (1985) 207-258.

[26] K. Rani, V. Rao, Control of fermenters - a review, Bioprocess Engineering 21 (1999) 77-88.

[27] K.-Y. San, G. Stephanopoulos, Optimization of fed-batch penicillin fermentation: a case of singular optimal control with state constraints, Biotechnology and Bioengineering 34 (1989) 72-78.

[28] I. Smets, G. Bastin, J. Van Impe, Feedback stabilization of fedbatch bioreactors: non-monotonic growth kinetics, Biotechnology Progress 18 (5) (2002) 1116-1125.

[29] I. Smets, D. Dochain, J. Van Impe, Optimal temperature control of a steady-state exothermic plug flow reactor, AIChE Journal 48 (2) (2002) 279-286.

[30] I. Smets, J. Van Impe, Optimal control of (bio-)chemical reactors: generic properties of time and space dependent optimization, Mathematics and Computers in Simulation 60 (6) (2002) 475-486.

[31] J. Staniškis, D. Levišauskas, An adaptive control algorithm for fed-batch culture, Biotechnology and Bioengineering 26 (1984) 419-425.

[32] S. Szépe, O. Levenspiel, Optimal temperature policies for reactors subject to catalyst deactivation-I. Batch reactor, Chemical Engineering Science 23 (1968) 881-894.

[33] J. Van Impe, Modeling and optimal adaptive control of biotechnological processes, Ph.D. thesis, Departement Electrotechniek, Faculteit Toegepaste Wetenschappen, Katholieke Universiteit Leuven, Leuven, Belgium, 1993.

[34] J. Van Impe, G. Bastin, Optimal adaptive control of fed-batch fermentation processes with multiple substrates, in: Proceedings of the 2nd IEEE Conference on Control Applications, Vancouver, Canada, 1993.

[35] J. Van Impe, G. Bastin, Optimal adaptive control of fed-batch fermentation processes, Control Engineering Practice 3 (7) (1995) 939-954.

[36] J. Van Impe, G. Bastin, Optimal adaptive control of biotechnological processes, in: J. Van Impe, P. Vanrolleghem, D. Iserentant (Eds.), Advanced Instrumentation, Data Interpretation, and Control of Biotechnological Processes, Kluwer Academic Publishers, Dordrecht, Boston, London, 1998, pp. 401-436.

[37] J. Van Impe, B. Nicolaï, P. Vanrolleghem, J. Spriet, B. De Moor, J. Vandewalle, Optimal control of tbe penicillin $G$ fed-batch fermentation: an analysis of a modified unstructured model, Chemical Engineering Communications 117 (1992) 337-353.

[38] T. Yamané, M. Kishimoto, F. Yoshida, Semi-batch culture of methanol-assimilating bacteria with exponentially increased methanol feed, Journal of Fermentation Technology 54 (4) (1976) 229-240. 